\title{
Reply to "Comment on
}

\section{'Alternative approach to the solution of the dispersion relation for a generalized lattice Boltzmann equation"" by P. Lallemand and L.-S. Luo}

\author{
T. Reis \\ Department of Mathematics, University College, London, WC1E 6BT, UK \\ T. N. Phillips \\ School of Mathematics, Cardiff University, Cardiff, CF24 4AG, UK
}

\begin{abstract}
In this reply to the comment by Lallemand and Luo, we defend our assertion that the alternative approach for the solution of the dispersion relation for a generalised lattice Boltzmann dispersion equation presented in [1] is mathematically transparent, elegant, and easily justified. Furthermore, the rigorous perturbation analysis used in [1] does not require the reciprocals of the relaxation parameters to be small.
\end{abstract}


In this reply, we respond to the comment by Lallemand and Luo on our recent article on an alternative approach to the solution of the dispersion relation for a generalised lattice Boltzmann equation [1].

Although there is a definite similarity between the method described in detail in [1] and that described by Luo et al. [2], there is also a very important difference. The collision operator $\mathbf{J}$ introduced by Luo et al. [2], which is related to the evolution operator $\mathbf{H}$ as follows:

$$
\begin{aligned}
\mathbf{H} & =\sum_{n=0}^{\infty} \frac{1}{n !} \delta_{t}^{n} \mathbf{H}^{n}, \\
\mathbf{H}^{n} & =\mathbf{K}^{n}(\mathbf{I}+\mathbf{J}), \\
K_{i j} & =-\imath \mathbf{c}_{i} \cdot \mathbf{k}_{m} \delta_{i j},
\end{aligned}
$$

is circulant and does not introduce the unknown constants associated with the generalised lattice Boltzmann equation (GLBE) that need to be determined.. Therefore, the eigenvalues of the matrix system in [2] can be written down using the well-known properties and theorems of circulant matrices and the system does not require the manipulation needed for general non-Hermitian matrices. The extension of the analysis to the GLBE case is not trivial since the Jacobian $\mathbf{J}$ satisfies different properties. The general form of the eigenvalues is not known for the GLBE. The calculation of the eigenvalues of relatively simple evolution operators $\mathbf{H}$ can also be performed with mathematical software such as Maple in a relatively straightforward fashion. However, direct computation of the eigenvalues of more complex operators $[1,3]$ becomes very complicated due to the seven unknown constants and six relaxation parameters and may be beyond the available computing resource capabilities. Therefore, a full and complete account of the algebraic and mathematical details, such as that presented in Reis and Phillips [1], is important in order to facilitate future LBE dispersion studies on more detailed lattices and the development of further advanced models.

Lallemand and Luo question our own claims about the validity of the perturbation method at large Knudsen numbers. In theory, the perturbation approach presented in Reis and Phillips [1] is valid for all Knudsen numbers $K n$ - it is the LBE itself that is limited to small Knudsen numbers $(K n<1)$. Indeed, the perturbation approach to the solution of the dispersion equation is valid of all $K n$ within the LBE regime. When linearising the LBE we write $N_{i}=N_{i}^{(e q)}+\varepsilon N_{i}^{(1)}+\cdots$, where $\varepsilon$ is the Knudsen number which is required to be small. In this well-known formulation, it is clear that $N_{i}$ departs only slightly from its 
equilibrium solution. All subsequent comments made by Lallemand and Luo with regard to the Knudsen number, in their Comment, highlight the shortcomings of the LBE and not the perturbation method itself.

We would also like to emphasise that the Reis and Phillips article [1] does not pretend to recover anything other than the Navier-Stokes equations. Instead it presents a clear and systematic approach to solving a mathematically difficult problem. We fully agree that the original paper of Lallemand and Luo [3] is an excellent piece of theoretical science and an important addition to the lattice Boltzmann field but nevertheless their chosen approach to solving the dispersion equation is not, from a mathematical viewpoint, the most transparent or most obviously justified. Moreover, the restriction implied in Lallemand and Luo [3] (that the relaxation parameter - which is related to the Knudsen number - has to be bigger than the wave vector $\mathbf{k}$ ) is not needed in Reis and Phillips [1]. Regarding the 'redundant' claim with respect to the Chapman-Enskog analysis, we accept that the language may be a little dramatic but we would like to add that this is the philosophical point which was first implied by Lallemand and Luo [3]. In their paper, they argued that if the hydrodynamic modes behave in exactly the same way as those of the linearised Navier-Stokes equations ( up to a certain order of $\mathbf{k}$ ) then the LBE is indeed adequate to simulate the Navier-Stokes equations.

The expressions for the transport coefficients derived in [1] are identical to those in [3] and it has never been claimed otherwise. In fact, the agreement of the expressions for the transport coefficients derived using the perturbation analysis [1] and the Gaussian elimination method [3] is a useful validation exercise and adds further support to the science. The Reis and Phillips paper offers only one criticism of Lallemand and Luo's theory, that is the Gaussian elimination used in [3] relies on the quantity $1 / s_{\alpha}$ being small. The results in both papers show that $0<s_{\alpha}<2$, which is not fully consistent with Lallemand and Luo's initial assumption and therefore questions their methodology. We would argue, from a purely mathematical viewpoint, that this is a weakness in their approach.

Reis and Phillips [1] have not sought to undermine the importance of the work by Lallemand and Luo [3] - the significance of their contribution to the field is fully appreciated and understood, and has been acknowledged. The aim was to present an alternative procedure for solving the lattice Boltzmann dispersion equation using a rigorous perturbation analysis 
that is mathematically transparent, elegant, and easily justified.

[1] T. Reis and T. N. Phillips, Phys. Rev.E 77, 026702 (2008).

[2] L. Luo, H. Chen, S. Chen, G. Doolen, and Y. Lee, Phys. Rev. A 43(12), R7097 (1991).

[3] P. Lallemand and L. Luo, Phys. Rev. E 61, 6546 (1999). 